\title{
HIDDEN MENACE: RECOGNIZING AND CONTROLLING THE HAZARDS POSED BY SMALLER AND LOWER POWER LASERS
}

\author{
Paper \# 1303
}

\author{
Samuel M. Goldwasser ${ }^{1}$ and Ben Edwards ${ }^{2}$ \\ ${ }^{1}$ Laser Teaching Center, Stony Brook University, Stony Brook, NY, 11794, USA \\ ${ }^{2}$ Duke University Medical System, Durham, NC, 27710, USA
}

\begin{abstract}
The hazards of large high power lasers are well recognized, and the necessity for controlling these hazards justifiably enjoys wide consensus. However, physically small lasers, or those with relatively low output power (e.g. Class 3B), can also present significant safety issues. The safety aspects of such small or lower power lasers often suffer from neglect because of the tendency to downplay, ignore, or simply fail to recognize the associated hazards. This work will assist safety professionals in identifying and articulating these "harder to sell" hazards by reviewing the specific issues (e.g. invisible laser radiation, potential for electrical shock, and even thermal/fire damage to materials), as well as providing some specific examples of readily available lasers for which these hazards exist. Finally, this paper addresses controlling those hazards through better recognition of the laser technology involved, and by proper work habits to minimize the chances of accidents and injuries associated with such laser systems
\end{abstract}

\section{Introduction}

It is well known that high power and generally physically large lasers pose hazards in many areas, including the potential for instant permanent damage to vision, burning of flesh or other materials, and when servicing or even adjusting internal optics, the possibility of shock or electrocution, among many other hazards. Less appreciated is the fact that much smaller laboratory lasers, hand-held lasers, or even lasers in a package the size of a pea, can have many of these same hazards, as well as other concerns that are not obvious. For example:

- $\quad$ Diode pumped solid state (DPSS) green (532 $\mathrm{nm}$ ) lasers and laser pointers may produce a much higher output power than the value specified by the manufacturer, as well as a dangerously large amount of infrared (IR) radiation at 1,064 $\mathrm{nm}$. This occurs mostly with inexpensive imported laser pointers, but also with some laboratory lasers.

- In addition to potential IR leakage, high power handheld DPSS lasers are now available with a small size and portability that may diminish the user's appreciation of the hazard involved. At least one recent eye injury is attributed to just such a high power handheld device.

- Various types of laser diodes the size of a grain of sand can output over 1 watt at various visible and near-IR wavelengths including $445 \mathrm{~nm}$ and $808 \mathrm{~nm}$, and be powered from AA cell batteries.

- Small flashlamp pumped solid state lasers may operate at 1,000 V or more, with tens of joules internally in energy storage capacitors, which may be exposed during alignment or other service. These may also be battery powered.

- Small DPSS lasers may have the alternating current (AC) line-connected switched-mode power supply in the same case as the low voltage controller/driver, fully exposed during adjustments or servicing.

- The ubiquitous helium-neon laser, with its high voltage (HV) power supply and tube, can store a considerable electrical charge after being disconnected from the AC outlet. This stored charge, while insufficient to kill, may induce a jerk reflex that causes minor injury or other damage (e.g. dropping the laser on the floor).

For new lasers purchased from a reputable manufacturer, most of these risks will be well detailed in the operator's manual. However this valuable information is only helpful if users take the time to read it. Regrettably the tendency of many laser users is to skip over the chapter on safety so as to get the new laser installed and operational as quickly as possible.

Less reputable suppliers (e.g. low cost, and even not so low cost, lasers from Far East manufacturers) tend to 
provide little or no information on safety. These stripped down devices may also lack critical safety features - even those nominally required by the U.S. government's Food and Drug Administration Center for Devices and Radiological Health.

Surplus dealers, resellers, eBay, and others provide a growing number of used lasers, often with no manual or specification documentation at all. Furthermore, there's always a definite possibility that a used laser may either be defective or modified in some way not disclosed to the buyer. Such defects or modifications may compromise safety, e.g. IR-blocking filter absent or interlocks bypassed.

Potential laser users must therefore be aware of the various safety issues associated with any laser they obtain from any source.

\section{IR Leakage from DPSS Visible Lasers}

A substantial amount of IR leakage from the pump diode and fundamental lasing process presents the most likely fault in visible (e.g., green) DPSS lasers. This may arise from a combination of imperfect mirror coatings that allow some IR through and/or an incorrectly oriented potassium-titanyl-phosphate (KTP) or other doubling crystal. The doubling crystal may even have broken loose, resulting in no visible output at all but substantial IR emission. Normally, there should be an IR-blocking filter or some other means of preventing substantial power at IR wavelengths from reaching the output aperture.

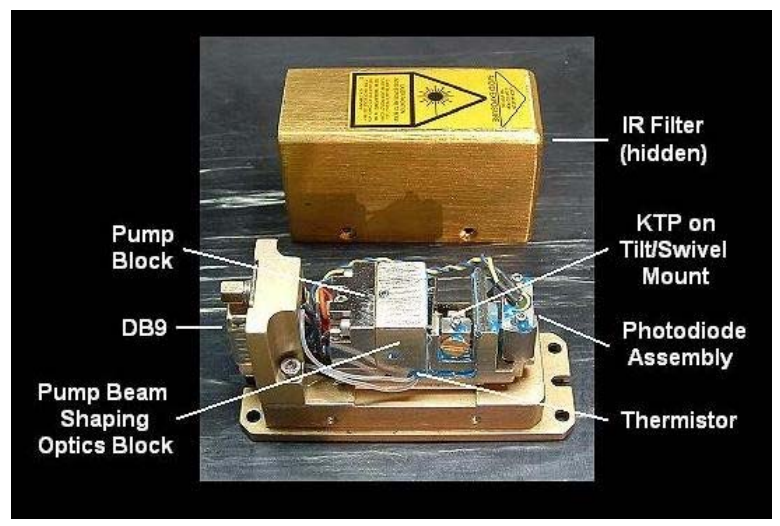

Figure 1 - JDS Uniphase uGreen laser head with IRblocking filter in cover

This small and very popular DPSS laser (Fig. 1) has its IR-blocking filter glued inside the cover, so when that is off for any reason (4 screws), the beam will have a substantial IR component. The filter could also have been removed due to damage (e.g., chemical attack damaging its surface) without affecting the laser's behavior in an obvious way.

A variety of techniques may be used to determine if any given laser is outputting enough IR to present a hazard. The following methods assume a visible wavelength DPSS laser.

- IR Viewing Card: generally only show visible wavelengths (i.e., green) if it is pure; IR will appear as a red or orange glow surrounding it.

- Comparison - Silicon versus Thermal Power Meter: If two types of calibrated laser power meters are available, a major discrepancy between their readings indicates that multiple wavelengths are present.

- Diffraction Grating and Digital Camera: Galang et al (2010) [1] describe a "kitchen table" setup that will identify IR wavelengths using a $\mathrm{CD}$ as a diffraction grating and a Webcam or other digital camera with its IRblocking filter removed.

- Monochromator, Optical Spectrum Analyzer (OSA), or Spectrometer: Any of these instruments that cover the near-IR (it is not necessary that the instrument also detect the visible wavelengths) will easily pick up both the fundamental and pump IR (i.e., $808 \mathrm{~nm}$ and $1,064 \mathrm{~nm}$ for a $532 \mathrm{~nm}$ green DPSS laser).

- Scanning Fabry-Perot Interferometer (SFPI): When used with a mirror set that is optimal for the visible wavelength, IR will either show up as broad ripples rather than sharp narrow peaks, or will overwhelm and saturate the sensor making a display of the laser impossible. Where little or no IR is present, the display will be normal.

- Measurements with an Added IR-Blocking Filter: When using a laser power meter to take readings with and without an external IRblocking filter, they should differ by no more than a few percent depending on the type of filter and any AR coatings that may be present.

- Internal Inspection: If the laser isn't sealed, looking inside will usually reveal whether an IR-blocking filter is present. It would likely have the appearance of green or blue glass and appear as one of the last optics before the output aperture unless IR is suppressed by transmission in internal turn mirrors (e.g. Coherent 215M, $315 \mathrm{M}$, and 415M lasers), in which case 
anything that impacts IR leakage will likely also affect the normal laser output.

Total suppression of IR is not possible, but an acceptable value would be below perhaps 0.1 percent of the design wavelength's output, not tens of percent.

\section{High Power Hand-Held Laser "Pointers"}

Wyrsch et al [2] report an eye injury to a 15-year old boy who purchased a $150 \mathrm{~mW}$ "laser pointer" for use as a toy (e.g. for popping balloons). The injury occurred as the boy played with the laser in front of a mirror to create a "laser light show", striking his eyes several times with the beam. This laser had dimensions and appearance very similar to the generally innocuous $5 \mathrm{~mW}$ laser pointers in common use for decades. As those authors concluded:

"Neither the owners nor the potential victims of such dangerous 'toys' can distinguish harmless laser pointers from hazardous ones, and we may see more such eye injuries in the near future.”

In addition to high power lasers disguised as relatively harmless $5 \mathrm{~mW}$ laser pointers, several manufacturers (e.g. Laserglow Technologies, Wicked Lasers, Dragon Lasers, etc.) also offer completely portable handheld lasers the size of a flashlight, with output powers of well over one watt. Again, the small size, relatively low cost, unrestricted availability, and complete mobility of these devices conspire to both decrease the perceived hazard and increase the difficulty in implementing safety control measures. Furthermore, the direct marketing of these products to the general public means that recreational buyers completely bypass the workplace safety infrastructure that normally protects institutional users of lasers for industrial, research, medical, and educational purposes.

A Laser Institute of America (LIA) press release [3] in late August 2010 warned of the dangers posed by a one watt portable laser selling for $\$ 300$. That release urged that any such Class 4 lasers should not be purchased unless the buyer has had appropriate laser safety training and an understanding of how to secure such devices within a Class 4 laser controlled area. Laser safety professionals should join LIA in discouraging the recreational use of Class $3 \mathrm{~b}$ and 4 lasers, and consider options for curtailing the unrestricted distribution of these inexpensive high power handheld lasers to untrained members of the general public.

\section{Small High Power Laser Diodes}

High output power from small lasers mostly applies to laser diodes (LD), but common hand-held green laser pointers can also represent a potentially very serious hazard. Even if not sold as high power, some can be boosted to over $100 \mathrm{~mW}$ by changing an internal resistor. While unlikely, this can also happen due to a part failure. Also, many pointers sold as having a 5 $\mathrm{mW}$ maximum have power fluctuations resulting in much higher than $5 \mathrm{~mW}$ appearing at times as thermal conditions change. (For technical reasons, red laser pointers are generally not susceptible to being boosted as dramatically either deliberately or by accident.)

The variety of high power LDs now readily available from many sources pose a greater concern. Their small size and modest electrical power requirements make them appear deceptively innocuous when in fact the amount of optical output power is enough to melt some materials, ignite combustible items, and cause instant eye injury, especially if coupled with a simple collimating lens (e.g. from a DVD player). The output of IR LDs is nearly or totally invisible, making them much more dangerous. Schmitz [4] describes the components of Casio projectors, from which the now common $445 \mathrm{~nm}$ (deep blue) $1 \mathrm{~W}$ laser diodes (Fig. 2, $3)$ can be easily scavenged. The perceived brightness, and hence perceived risk, of these lasers is diminished by the human eye's relatively low sensitivity at that wavelength (about 1/25th the sensitivity at the $555 \mathrm{~nm}$ peak). The small package size (5.6 mm can) and low cost of these LDs further diminish awareness of the hazard. Other high power IR LDs may be packaged on a bare $5.6 \mathrm{~mm}$ header, closed in a 5.6 or $9 \mathrm{~mm}$ can, a C-mount, or other configurations, and may produce several watts of output.

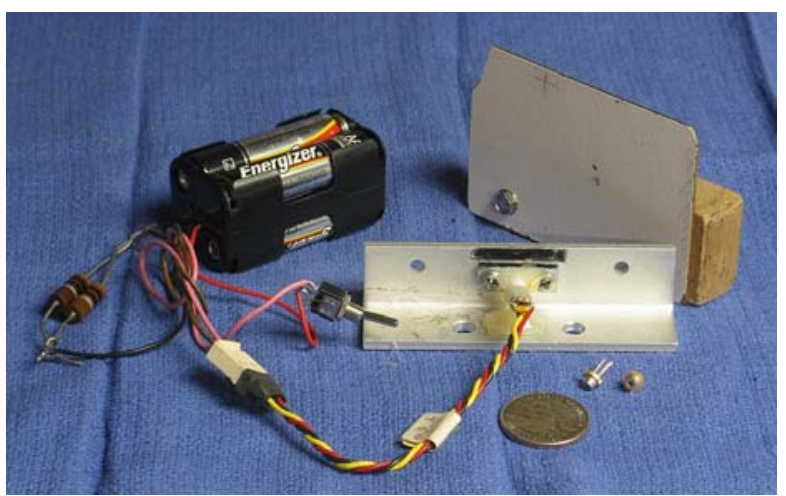

Figure 2 - One watt $445 \mathrm{~nm}$ laser diode from Casio projector, powered by AA Cells

While cooling is required to run for an extended period of operation, the power for the LD itself can be provided by batteries (even a single AA cell with a boost driver) or a common DC wall adapter. The fact that many LDs are very susceptible to damage from improper power or electrostatic discharge doesn't make them any less dangerous. 


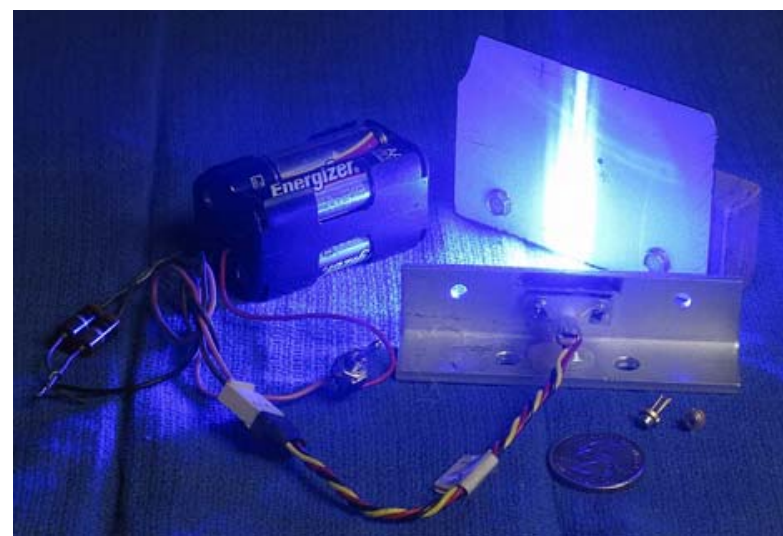

Figure 3 - Output from one watt $445 \mathrm{~nm}$ laser diode from Casio projector

The electronics used to drive the one watt LD from the Casio projector (Figs. 2 and 3) consists of 4 AA cells, a pair of $10 \mathrm{ohm}$ resistors, and a power switch. The LD is mounted in a small heat-sink with no collimator. A U.S. quarter is shown along with 2 bare similar LDs for size comparison. In Fig. 3, the LD is running at about $100 \mathrm{~mW}$, limited only by the values of the series resistors.

The initial testing of LDs may be accomplished by using a thermal power meter and monochromator, OSA, or spectrometer to triage any unidentified laser diodes when first powering them. Once the output wavelengths are known, an appropriate semiconductor or thermal power meter can be used if desired to establish the LD's output power range.

Treat laser diodes like any other high power laser regardless of their physical size, especially if coupled to any collimating or focusing optics. Use an appropriate beam stop and eye protection.

\section{Small Pulsed Solid State Lasers}

These are typically neodymium:yttrium-aluminumgarnet (Nd:YAG), erbium:YAG (Er:YAG), or similar types of lasers and may include a passive or active Qswitch. Examples include the SSY1 [5], Kigre MK-367 [6], and similar military surplus lasers [7], semiconductor mask trimmers, and many others. The actual laser head is deceptively compact in many of these - 1x1x4 inches for SSY1 (Fig. 4). The power supply can be similarly compact.

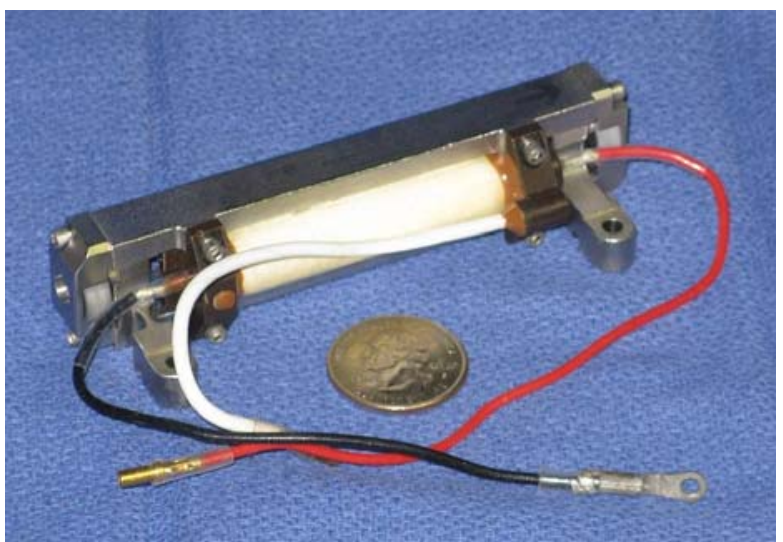

Figure 4 - Military surplus SSY1 pulsed Q-switched solid state laser head

These have all of the vision dangers associated with pulsed lasers, despite their small size. For example, when the SSY1 is coupled with its stock pulse forming network (PFN1), it may produce anywhere from a few $\mathrm{mJ}$ to $25 \mathrm{~mJ}$ or more in a several ns pulse. This is enough to punch holes in aluminum foil. PFN1's capacitor is capable of storing over $15 \mathrm{~J}$ of electrical energy at $900 \mathrm{~V}$, which is a substantial fraction of what is considered likely to be lethal under the wrong conditions. And on a home-built system, electrical connections may not be as well insulated as needed. Furthermore, some users have replaced the PFN1's energy storage capacitor with one storing over $500 \mathrm{~J}$, rendering the SSY1 capable of over $1 \mathrm{~J}$ of output and the ability to blast holes in razor blades, as well as instant electrocution should one come in contact with the high voltage terminals.

The military surplus M60 tank rangefinder laser (Fig. 5) is somewhat larger than the SSY1, with the lasing rod being 3 inches in length and the head about 8 inches overall. Like the SSY1, this rangefinder too has been readily available through the low cost surplus market. The capacitor bank shown in the photo is capable of storing a very lethal charge of almost 1,000 Joules at 1,400 V. Two such capacitor banks were purchased on eBay for about \$20 total including shipping.

Appropriate control measures must be implemented for all Class $3 \mathrm{~b}$ and 4 pulsed lasers, including physically small devices. Aftermarket surplus laser users should incorporate sufficient engineered controls to bring these devices into compliance with the requirements of the U.S. Federal Laser Product Performance Standard (Title 21 Code of Federal Regulations 1040.10). 


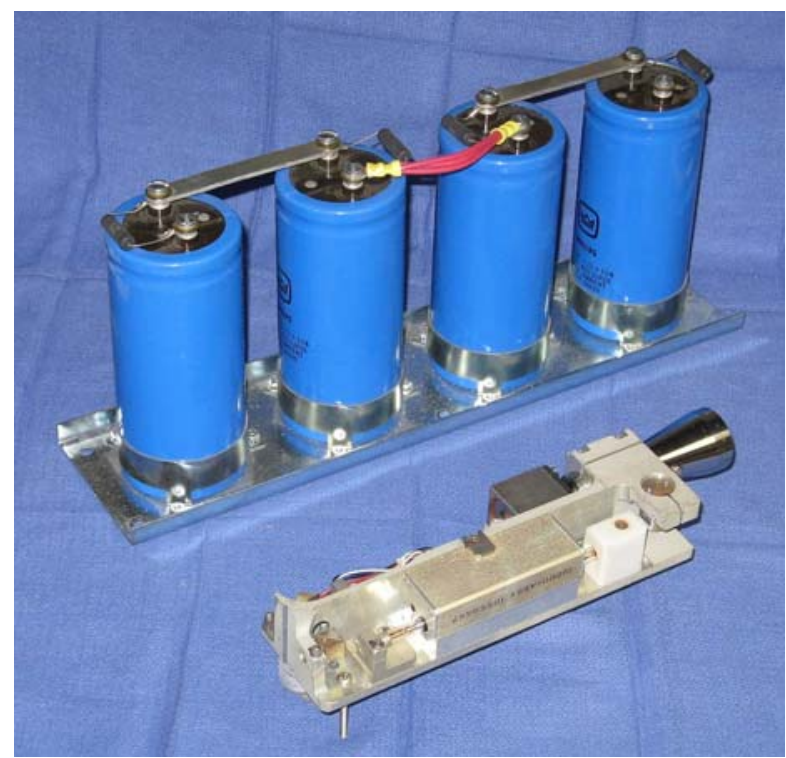

Figure 5 - Military surplus pulsed Q-switched solid state laser head with energy storage capacitor bank

\section{General Electrical Hazards}

In the early days of lasers, virtually all systems were potentially lethal since most were either pulsed solid state lasers with large HV capacitors or gas lasers with HV power supplies. But even modern DPSS lasers running on low voltage direct current (DC) can pose risks of electric shock due to the $\mathrm{HV}$ alternating current (AC) line-connected switched-mode power supply (SMPS) that may be present in the same box as the controller (Fig. 6). While not an issue during normal use, one may be motivated to attempt adjustments to peak performance to the DPSS driver and come in contact with exposed HV terminals, especially as such adjustments may often be performed in subdued lighting.

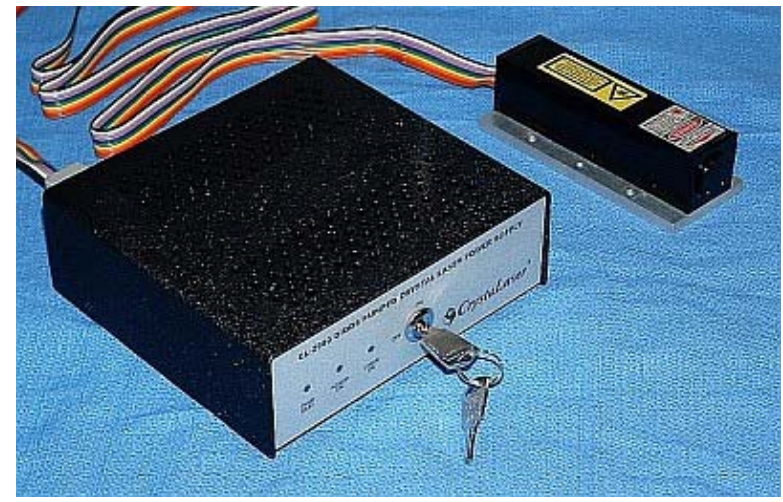

Figure 6 - Crystalaser DPSS laser system and controller

While the AC line-connected switched-mode power supply has most HV terminals reasonably well protected, there are still some that are exposed that could be accidentally touched while making adjustments to the DPSS driver on the left (Fig. 7).

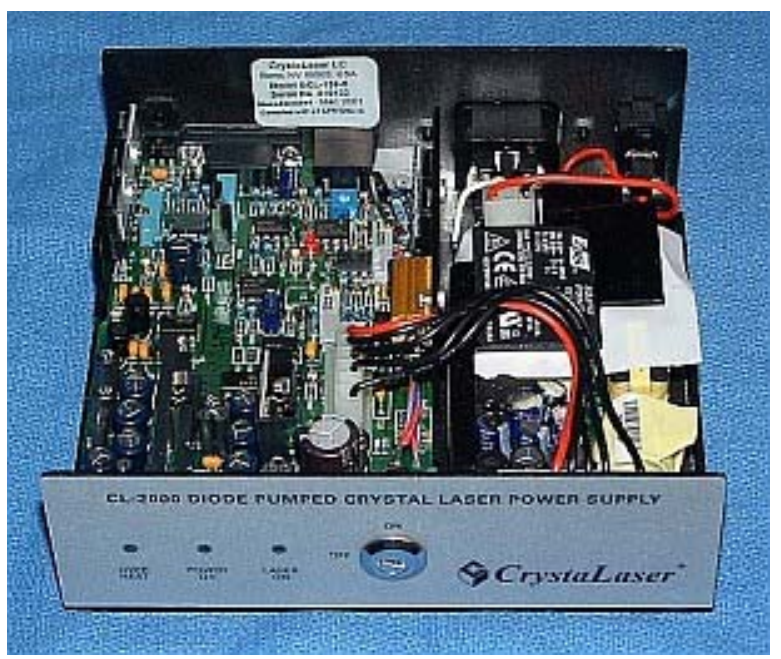

Figure 7 - Crystalaser DPSS laser controller showing SMPS next to DPSS driver printed circuit board

Even the ubiquitous helium-neon (HeNe) laser isn't exempt from electrical hazards. HeNe lasers do run on $\mathrm{HV}$ power - typically between $1 \mathrm{kV}$ and $5 \mathrm{kV}$ at 3 to $12 \mathrm{~mA}$. The typical HeNe laser consists of a laser head (shown below) and separate power supply, attached via an "Alden" connector (Fig. 8).

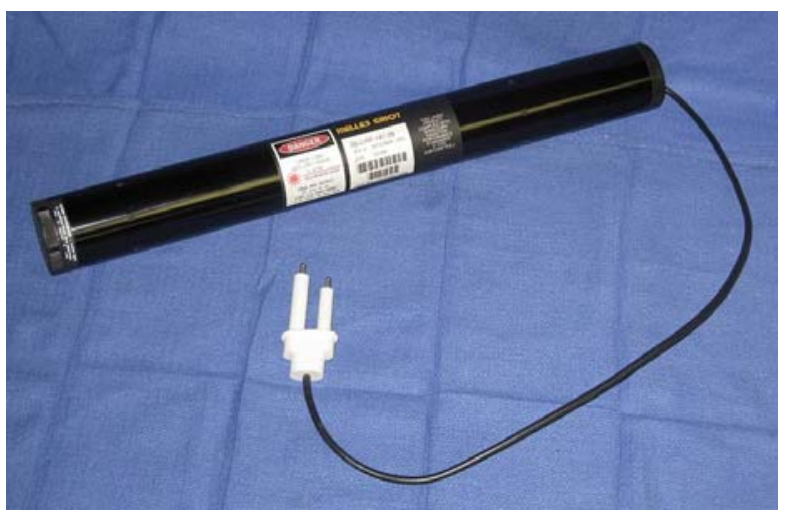

Figure 8 - Modern HeNe laser head with high voltage (“Alden”) connector

The HV negative return often runs through the metal laser head and with some, only a simple easily damaged spring contact completes the circuit to the cable. Should it cease to make good contact, the entire cylinder in the photo becomes electrically live. While not likely to be lethal, the collateral damage from being startled and dropping the laser can be annoying. Another hidden hazard comes about if for some reason the unit is powered up but the tube does not start. This is common with older or high mileage HeNe lasers, especially if they haven't been used in awhile. But if 
this happens and the laser head is disconnected from the power supply, the capacitance of the cable and tube store a painful, if not really dangerous, charge at voltage of up to $10 \mathrm{kV}$ or more for a long time. If the shorter prong is touched while holding onto the cylinder, the results can be unpleasant, and dropping the laser will again be the likely result.

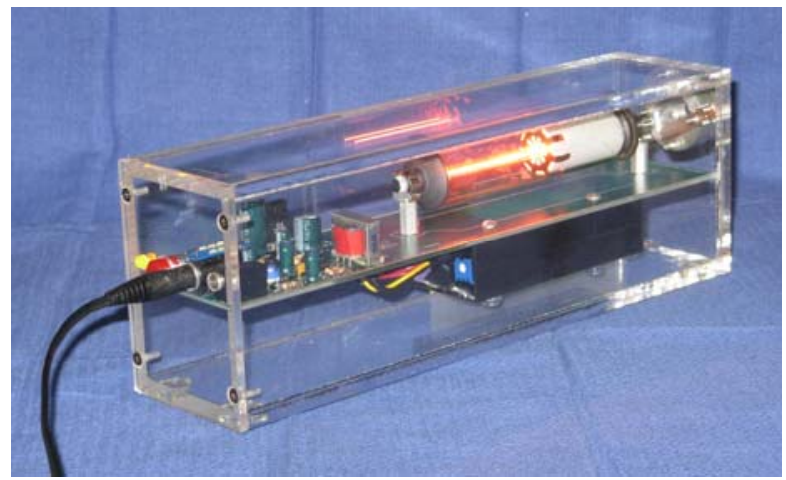

Figure 9 - Demo HeNe laser in clear case.

This demonstration "visible" HeNe laser (Fig. 9) runs from a 12 VDC wall adapter and has no HV warnings anywhere. With the Plexiglas cover, everything is safely enclosed. But should the inner assembly be removed for any reason, the HV anode of the tube (near the center) is fully exposed, asking to be touched.

\section{Conclusions}

For a new laser - regardless of size or power, READ the entire accompanying manual, paying special attention to the chapter on SAFETY. If there is no manual or one that's not useful, treat the laser like one that's used, surplus, or may have been modified, and take the time to not only understand the basics of how they are supposed to work and are constructed, but to also carefully inspect the laser and controller/power supply for potential optical and electrical dangers.

Fully implement generally recognized control measures for each Class 3B or 4 laser. Until permanently mounted in an experiment or other instrument with a fully confined beam, don't overlook the well known safety guidelines, including those for laser safety eye wear - mandatory for pulsed or invisible wavelength lasers!

This list of hazards hidden in small and lower power lasers is of course incomplete, and safety professionals must remain vigilant in identifying, assessing, and controlling other hidden dangers. Two obvious trends have dominated the development of laser designs: the size and cost needed to achieve a given output power have both steadily declined over time. As laser technology continues to evolve and more novel applications emerge, laser users and the safety staff who support them must recognize and address not only the obvious concerns, but also the hidden menace.

\section{References}

[1] Galang J., Restelli A., Hagley E. W., Clark C. W. (2010) A Green Laser Pointer Hazard (NIST Technical Note 1668), National Institute of Standards and Technology.

[2] Wyrsch S., Baenninger P.B., \& Schmid M.K. (2010) Retinal injuries from a hand held laser pointer, N Engl J Med 363(11):1089 - 1091.

[3] Laser Institute of America (2010) 1 watt portable blue laser poses a hazard. Available on line: www.laserinstitute.org/news/2010/08/31/1-wattportable-blue-laser-poses-a-hazard/

[4] Schmitz E. (2010) Casio XJ-A 130-2450 - LED and laser instead of UHP! (product review). Available on line: www.cine4home.com/tests/3-tests/31-casioxja-1302450--led--laser-instead-of-uhp.html

[5] Goldwasser, S. M. (2010) A Small Nd:YAG Laser - SSY1, Sam's Laser FAQ, Available on line: www.repairfaq.org/sam/laserscl.htm\#sclsy1.

[6] Kigre Lasers (2010) Kigre high peak power permanently aligned MK-367 1,064 nm laser brochure. Available on line: www.kigre.com/files/mk367.pdf. [7] Goldwasser, S. M. (2010) Hughes rangefinder ruby laser assembly, Sam's Laser FAQ, Goldwasser, S. M. www.repairfaq.org/sam/laserscl.htm

\section{Meet the Authors}

Samuel M. Goldwasser has had extensive experience in both industry and academia and is an engineering consultant and author of "Sam's Laser FAQ" (http://www.repairfaq.org/sam/lasersam.htm),

generally considered the most comprehensive on-line resource for all types of laser information. He is also a visiting lecturer and mentor with the Laser Teaching Center at Stony Brook University, Stony Brook, NY (http://laser.physics.sunysb.edu/). Sam earned his B.S. from Drexel University, Philadelphia, PA, and M.S. and Ph.D. from the Massachusetts Institute of Technology, Cambridge, MA, all in electrical engineering.

Ben Edwards is a Certified Health Physicist with Duke University Health System's Division of Radiation Safety. He earned a BS degree in Physics and an MS in Occupational Safety. Ben has nearly two decades of experience as a radiation safety program manager and institutional laser safety officer. He is also a Certified Laser Safety Officer, Certified Medical Laser Safety Officer, member of the ANSI Z136 Committee, and currently serves as chair of the ANSI Z136 Non-Beam Hazards Technical Sub-Committee. 